\title{
Credence: A Belief-First Approach - Erratum
}

Andrew Moon and Elizabeth Jackson

DOI: 10.1017/can.2020.9, Published online by Cambridge University Press, April 27, 2020

In the original publication of this article (Moon and Jackson 2020) the multiplication sign was erroneously replaced with an addition sign in the following mathematical expression, which appeared four times on page 656 of the article: $567 \times 123=69,741$.

The publisher apologizes for the error.

\section{Reference}

Moon, Andrew and Jackson, Elizabeth. 2020. “Credence: A Belief-First Approach.” Canadian Journal of Philosophy 50: 652-669. DOI: $10.1017 /$ can.2020.9. 